\title{
Blood culture at 63 Japanese healthcare facilities
}

\author{
TAKAYUKI OHISHI ${ }^{1}$, MAYUMI OGAWA $^{2}$, ETSUKO KATSUKURA $^{1}$ and KAZUYA IMOTO ${ }^{1}$ \\ ${ }^{1}$ Department of Infection Control and Prevention, Saiseikai Yokohamashi Tobu Hospital, Yokohoma, Kanagawa 230-8765; \\ ${ }^{2}$ Department of Infection Control and Prevention, Saiseikai Hyogo Hospital, Kobe, Hyogo 651-1302, Japan
}

Received July 12, 2021; Accepted October 6, 2021

DOI: 10.3892/wasj.2021.131

\begin{abstract}
Blood culture is essential for the diagnosis of infectious disease. Appropriate treatment is administered according to blood culture results; thus, highly accurate tests are required. The present study obtained data from hospitals which are ranked among those with the highest number of beds in each prefecture (top 10\%; 1,000 hospitals nationwide), beginning with 3,129 hospitals throughout Japan with $\geq 200$ beds as of 2018. In total, 63 hospitals that gave their consent to participate in the study provided information regarding blood culture-related indicators over a 2-year period. The positive blood culture rate ( $95 \%$ confidence interval) was $15.4 \%$ (13.7-17.1\%), the number of blood culture sets per 1,000 patient days was 21.5 (18.2-24.9), multiple set collection rate was $76.7 \%(71.1-82.3 \%)$ and the contamination rate was $3.1 \%(2.4-3.8 \%)$. Using these results as reference values for blood culture in Japanese healthcare facilities with $\geq 200$ beds, the authors are attempting to approach these values at their resident hospitals and expect improved on-site interventions and educational activities regarding the state of blood culture.
\end{abstract}

\section{Introduction}

Blood culture is essential for the microbiological diagnosis of bacteremia, infective endocarditis, fever of unknown origin and other infectious diseases (1). The results of blood cultures are directly linked to appropriate treatment; therefore, the corresponding tests need to be highly accurate (2). The calculation and monitoring of the positive blood culture rate, the number of blood culture sets per 1,000 patient days, the multiple set collection rate and the contamination rate

Correspondence to: Dr Kazuya Imoto, Department of Infection Control and Prevention, Saiseikai Yokohamashi Tobu Hospital, 3-6-1 Shimosueyoshi, Tsurumi-ku, Yokohoma, Kanagawa 230-8765, Japan

E-mail: k_imoto@tobu.saiseikai.or.jp

Abbreviations: AL, alcohol; CHG-AL, chlorhexidine gluconate/ alcohol; PVP-I, povidone-iodine

Key words: blood culture, positive rate, set collection rate, multiple set collection rate, contamination rate (hereinafter referred to as 'the four indicators') have been proposed as indicators for the assessment of the suitability of blood culture (3-5). However, it is difficult to set international unified standard values as medical conditions differ between countries.

Japanese reference values must be calculated to assess the state of blood culture in a general hospital. Relevant Japanese studies include a field survey of blood culture in six Japanese healthcare facilities from 2007 to 2010 (6), a limited assessment of indicators in five healthcare facilities in 2014 (7), and limited studies by the All-Japan Hospital Association (8) and the Japan Hospital Association (9). Moreover, the Japan Surveillance for Infection Prevention and Healthcare Epidemiology (J-SIPHE), a joint infection control platform led by the Antimicrobial Resistance (AMR) Clinical Reference Center within the National Center for Global Health and Medicine, tabulated basic data that served as indicators (10). However, this report was not a stratified analysis that accounted for hospital size and therefore did not specifically demonstrate the four indicators according to hospital size.

A valid assessment of the suitability of blood culture in healthcare facilities requires clear domestic reference values by hospital size based on data from a multicenter study (6). Additionally, the positive blood culture rate differs based on blood samples drawn following antibiotic administration and on blood culture test systems (11). Furthermore, the antiseptic used when collecting blood for blood culture affects the culture contamination rates (12). Identifying the factors associated with these blood culture indicators may lead to more suitable blood collection protocols.

In the present study, in order to help improve the suitability of blood culture throughout Japan, information related to blood culture was obtained from $>200$ hospitals to investigate domestic reference values by hospital size. The findings of the present study may lay the foundation for the establishment of a standard protocol for the appropriate collection of blood in Japan and similar regions.

\section{Patients and methods}

Methods. The present study was a multicenter, retrospective, descriptive, epidemiological statistical study conducted from January, 2017 to December, 2018. Beginning with 3,129 hospitals throughout Japan with $\geq 200$ beds as of 2018 , hospitals were sampled from a corporate database (Landscape Co., Ltd.). Hospitals which are ranked among those with the highest 
number of beds in each prefecture (top 10\%; 1,000 hospitals in total, nationwide) were sampled and their cooperation with the study was requested. The hospitals provided information related to blood culture, excluding any personal information of patients.

Outcome measures. Outcome measures were based on a previous study (6). As the basic characteristics reflecting the attributes of healthcare facilities, the hospital size (number of beds), the total number of hospitalized patients by year and additional reimbursement obtained for infection prevention (hereafter termed 'additional reimbursement') paid by the Ministry of Health, Labor and Welfare of Japan when certain infection control standards are met (in other words, infection control is guaranteed to a certain standard) were surveyed. The total number of hospitalized patients (patient days) and the number of patients hospitalized every day at midnight were defined as their respective totals over a year.

The following data related to blood culture were collected in order to calculate the four indicators: The total number of sets, number of solitary sets, number of solitary sets in pediatric settings, number of positive sets, number of positive samples in only one set, the blood culture test system manufacturer and the antiseptics used for blood collection.

Positive blood culture rate. A positive blood culture rate indicates the suitability of blood collection timing for culture. A positive case was defined as a positive blood culture result in a solitary set, regardless of the bacterial strain detected. However, in the case that the same strain was detected in the same patient more than once within a period of 30 days (with the date of the first positive result defined as day 0), all detections from the second detection were considered invalid, and the case was defined as a single positive case. In the case that multiple strains of bacteria were detected in the same sample, each strain detected was defined as an independent case. The positive blood culture rate was calculated as follows: Positive blood culture rate $=$ number of positive sets/total number of collected sets $\mathrm{x} 100$.

Number of sets per 1,000 patient days. The number of sets per 1,000 patient days indicates whether blood culture sets were collected without failure from patients who required blood culture. This number was divided by the total number of hospitalized patients to minimize bias in set collection rates associated with the differences among hospitals in the number of hospitalized patients. This was calculated as follows: Number of sets per 1,000 patient days $=$ total number of collected sets/total number of hospitalized patients x1,000.

Multiple set collection rate. The multiple set collection rate is an indicator of accuracy management that contributes to the accurate diagnosis of bloodstream infections. A solitary blood culture set was defined as the sample obtained from a single venipuncture. The submission of multiple blood culture sets was defined as samples from multiple sets dispensed from multiple punctures on the same date; if the date was the same, the duration between set collections was irrelevant. Based on this definition, solitary set submission was defined as the collection of only one blood culture set on a given date (5). The multiple set collection rate was calculated as follows: Multiple set collection rate $=($ total number of collected sets - number of solitary sets)/total number of collected sets x100.

Contamination rate. The contamination rate indicates the education level of hospital staff members collecting blood and the condition of the blood collection environment. The contamination rate was defined as the total number of cases in which only one blood culture set of $\geq 2$ sets submitted on the same day tested positive for coagulase-negative staphylococci, Propionibacterium acnes, Micrococcus spp., Viridans streptococci, Corynebacterium spp. and Bacillus spp. (13). The contamination rate was calculated as follows: Contamination rate $=$ number of positive samples in only one set/(total number of collected sets - number of solitary sets) x100.

Statistical analysis. A descriptive epidemiological analysis was conducted for all values. The freely available EZR v1.50 software (Jichi Medical University, Saitama, Japan) was used for statistical analyses (14). To determine whether the means obtained from the samples were equal to the means of the total hospitalized population, a one-sample t-test was used and the $95 \%$ confidence interval $(95 \% \mathrm{CI})$ values were calculated.

Ethical considerations. The study protocol was approved by the Institutional Review Board of Saiseikai Yokohamashi Tobu Hospital (2018107). The present study: i) was an observational study that used only existing information and is not categorized under specified clinical research; ii) used existing specimen information; iii) was conducted solely by Saiseikai Yokohamashi Tobu Hospital; iv) did not involve any intervention; v) did not use human samples; vi) used anonymized sample information; vii) did not use medical history or other personal information requiring special care; viii) received sample information from other hospitals; ix) may have immense social significance; and $\mathrm{x}$ ) simplified explanations and consent. Informed consent was not required due to the retrospective nature of the study.

\section{Results}

The geographical status of the participating hospitals by prefecture is illustrated in Fig. 1. The 2-year total data for the four indicators are presented in Fig. 2. In addition, the data stratified by year and the number of beds are presented in Fig. 3. The number of sets per 1,000 patient days was affected by the total number of hospitalized inpatients and was therefore stratified by the total number of hospitalized patients rather than by the number of beds.

Status of participating facilities. Out of 3,129 facilities with at least 200 beds, 63 hospitals gave their consent to participate in the study. A total of 31 of the 47 prefectures of Japan were analyzed. The prefectures with the highest number of participating facilities were Hokkaido, Chiba, Tokyo and Kanagawa, with five facilities each (Fig. 1).

Basic characteristics of the participating facilities. The basic characteristics of the participating facilities are presented in Table I. The majority of facilities had obtained additional 


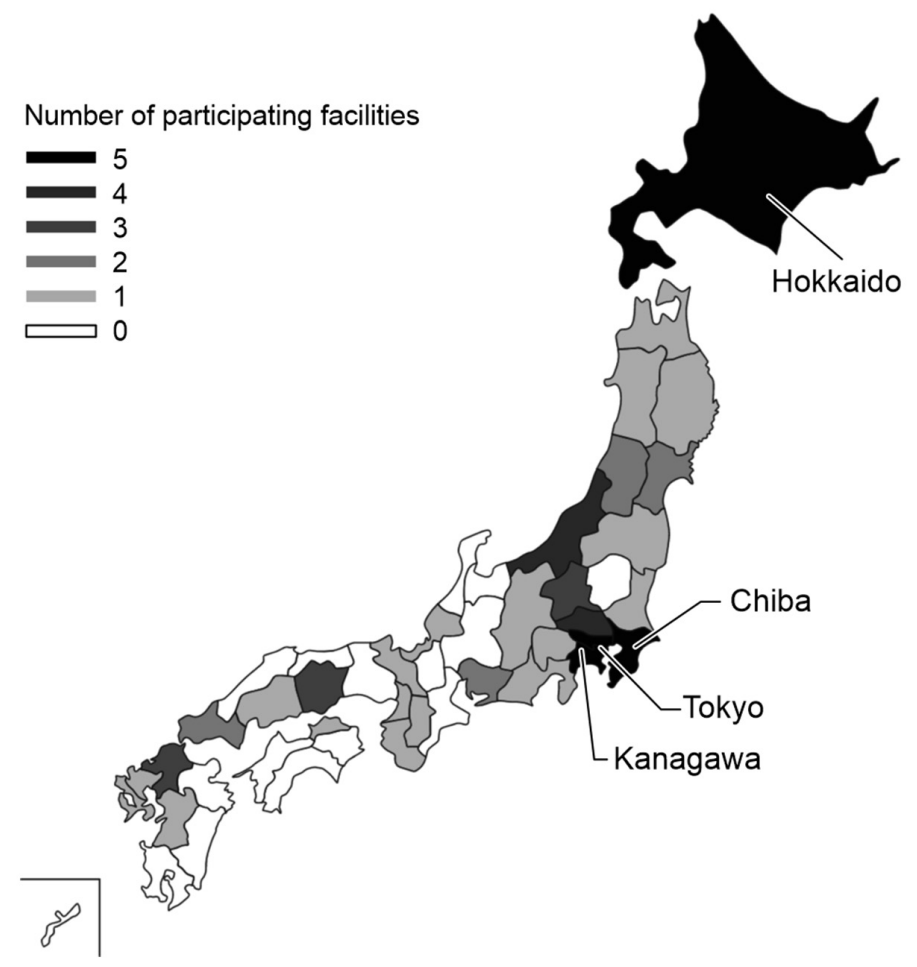

Figure 1. Status of the participating hospitals by prefecture.

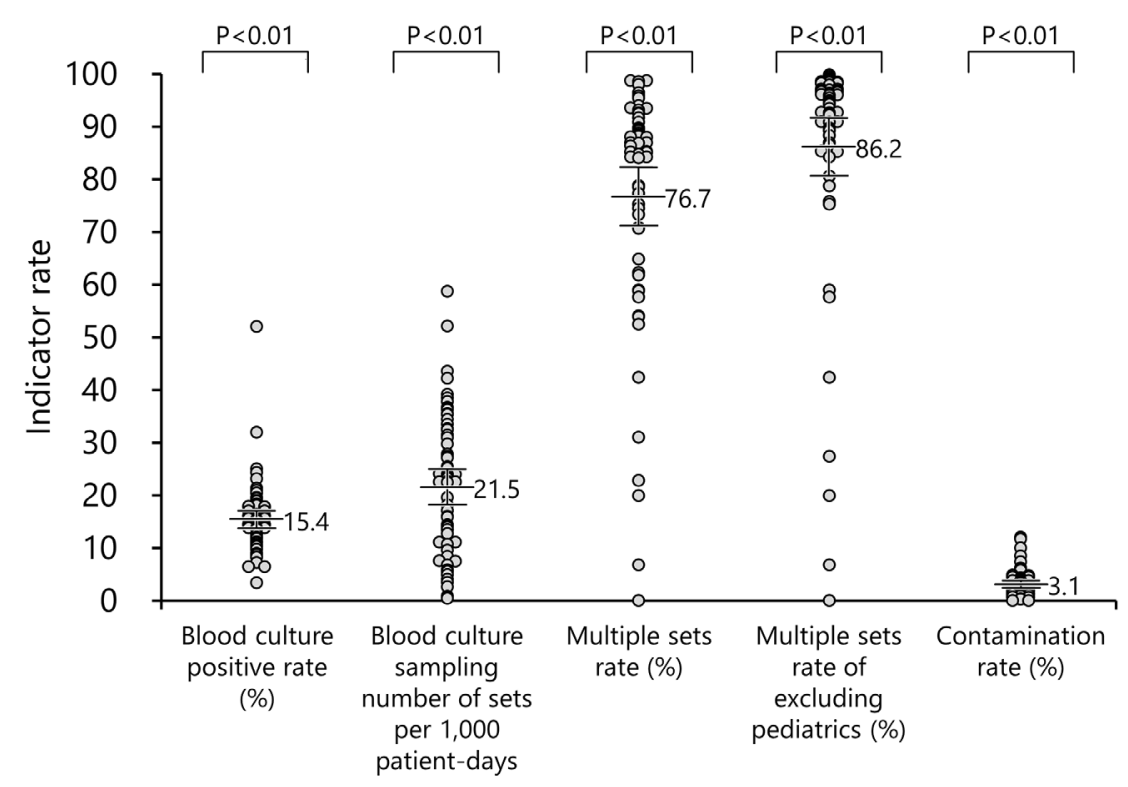

Blood culture indicators

Figure 2. Various blood culture indicators in 2017 and 2018 obtained from data provided by hospitals in Japan. The gray dots represent the values for each facility. The long bar represents the average of indicator rate. The error bar indicates the $95 \%$ confidence intervals.

reimbursement for the prevention of infection, which was granted by the Ministry of Health, Labour and Welfare when a hospital clears a certain standard related to the control of nosocomial infections. The total number of hospitalized patients was over eight million. The most common hospital size was 200-399 beds, whereas the number of hospitals with 400-499, $500-599$ and $\geq 600$ beds was identical. The smallest number of beds in any hospital was 200, whereas the largest number was 1,435 .
The most commonly used blood culture test system was from BD Biosciences, followed by bioMérieux and Beckman Coulter, Inc. The antiseptic most commonly used prior to blood collection was $10 \%$ povidone-iodine (PVP-I), followed by $1 \%$ chlorhexidine gluconate/alcohol (CHG-AL) and alcohol (AL).

Positive blood culture rate. The positive blood culture rate (95\% CI) during the study period was $15.4 \%$ (13.7-17.1\%, $\mathrm{P}<0.05$ ) (Table II). The hospital size with the largest mean 

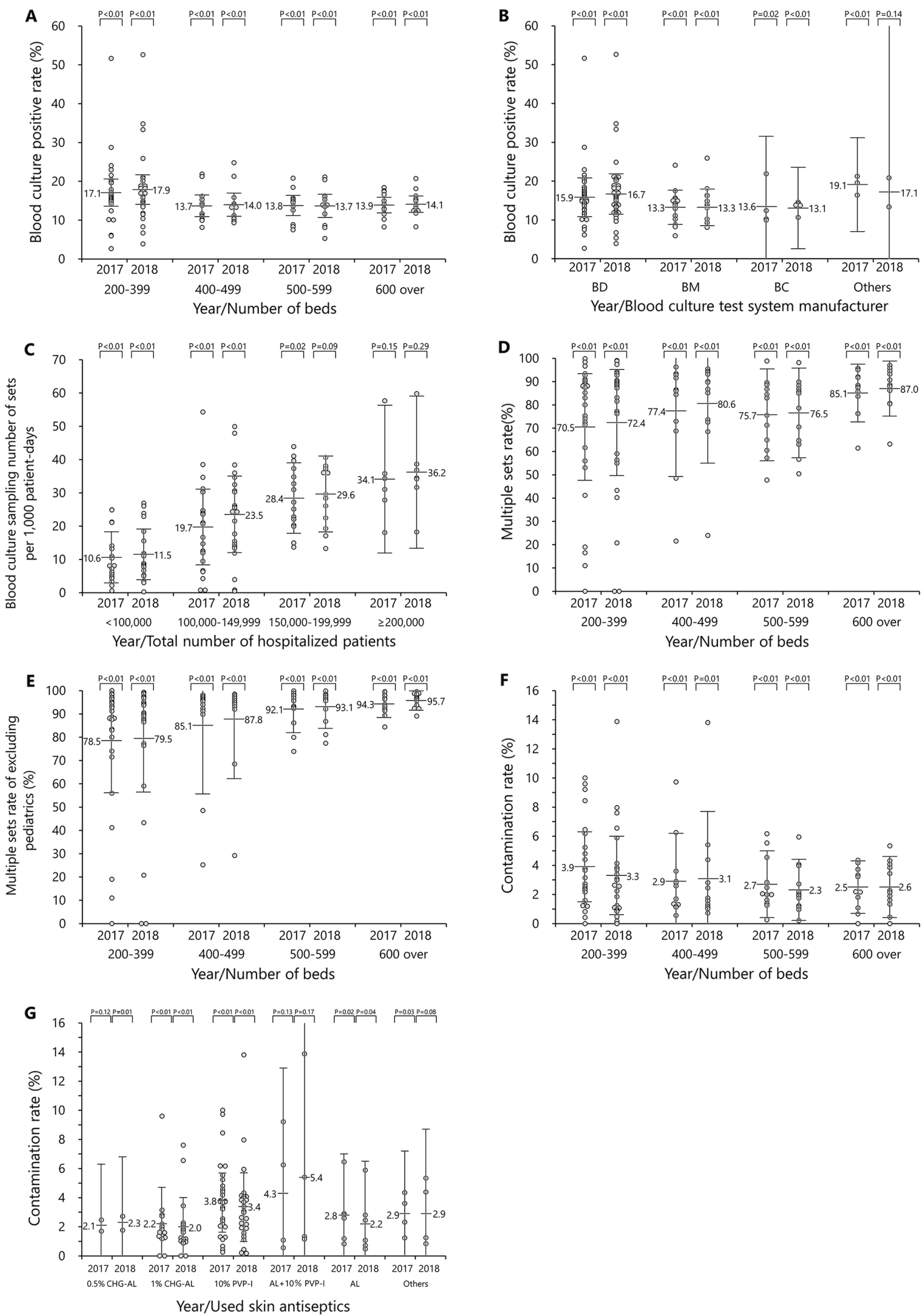

Figure 3. Blood culture-associated rates. (A) Positive blood culture rate per year, per number of beds. Gray circles represent medical facilities. (B) Positive blood culture per year, per blood culture test manufacturer. BD, BD Biosciences; BM, bioMérieux; BC, Beckman Coulter, Inc. (C) Blood culture sampling number per 1,000 patient days. (D) Multiple set collection rate per year, per number of beds. (E) Multiple set collection rate (excluding pediatric settings) per year, per number of beds. (F) Contamination rate per year, per number of beds. (G) Contamination rate per skin antiseptic. CHG-AL, chlorhexidine gluconate alcohol; PVP-I, povidone-iodine; AL+10\% PVP-I, 70\% alcohol, and 10\% povidone-iodine use. The results of each index will serve as a reference for blood cultures in medical facilities with $>200$ beds in Japan. The authors hope that on-site interventions and awareness-raising activities will be used in order to improve the blood culture status of each facility. The gray dots represent the values for each facility. The long bar represents the average of the indicator rate. The error bar indicates the $95 \%$ confidence intervals. 
Table I. Status of participating hospitals by prefecture.

\begin{tabular}{|c|c|c|}
\hline \multirow[b]{2}{*}{ Characteristic } & \multicolumn{2}{|c|}{ Year } \\
\hline & 2017 & 2018 \\
\hline \multicolumn{3}{|c|}{$\begin{array}{l}\text { Number of additional reimbursements for infection } \\
\text { prevention, no. }(\%)\end{array}$} \\
\hline First & $58(92.1)$ & $58(92.1)$ \\
\hline Second & $5(7.9)$ & $4(6.3)$ \\
\hline Unacquired & $0(0.0)$ & $1(1.6)$ \\
\hline \multicolumn{3}{|c|}{ Total no. of hospitalized patients } \\
\hline Number & $87,13,567$ & $86,81,663$ \\
\hline Average (range) & $1,38,310.6(61,304-464,314)$ & $1,37,804.1(57,533-472,534)$ \\
\hline \multicolumn{3}{|l|}{ No. of beds, no. (\%) } \\
\hline $200-399$ & $27(42.9)$ & $27(42.9)$ \\
\hline $400-499$ & $12(19.0)$ & $12(19.0)$ \\
\hline $500-599$ & $12(19.0)$ & $12(19.0)$ \\
\hline$>600$ & $12(19.0)$ & $12(19.0)$ \\
\hline \multicolumn{3}{|c|}{ Blood culture system manufacturer, no. (\%) } \\
\hline BD Biosciences & $39(61.9)$ & $41(65.1)$ \\
\hline bioMérieux & $17(27.0)$ & $17(27.0)$ \\
\hline Beckman Coulter, Inc. & $4(6.3)$ & $3(4.8)$ \\
\hline Others & $3(4.8)$ & $2(3.2)$ \\
\hline \multicolumn{3}{|c|}{ Recommended skin antiseptics } \\
\hline $0.5 \%$ CHG-AL, no. $(\%)$ & $2(3.2)$ & $2(3.2)$ \\
\hline $1 \% \mathrm{CHG}-\mathrm{AL}$ & $15(23.8)$ & $18(28.6)$ \\
\hline $10 \%$ PVP-I & $31(49.2)$ & $28(44.4)$ \\
\hline $\mathrm{AL}+10 \%$ PVP-I & $4(6.3)$ & $4(6.3)$ \\
\hline $\mathrm{AL}$ & $7(11.1)$ & $7(11.1)$ \\
\hline Others & $4(6.3)$ & $4(6.3)$ \\
\hline
\end{tabular}

CHG-AL, chlorhexidine gluconate alcohol; PVP-I, povidone iodine; AL + 10\% PVP-I, $70 \%$ alcohol and 10\% povidone iodine; AL, alcohol.

positive blood culture rate was 200-399 beds, with no marked differences among other hospital sizes (Fig. 3A). According to the blood culture test system manufacturer, the mean positive blood culture rate tended to be higher with BD Biosciences than with the other two manufacturers (Fig. 3B).

Sets per 1,000 patient days. The number of sets per 1,000 patient days $(95 \% \mathrm{CI})$ during the study period was 21.5 (18.2-24.9, $\mathrm{P}<0.05)$ (Table II). The mean number of sets among the total number of hospitalized patients increased proportionally with the total number of hospitalized patients (Fig. 3C).

Multiple set collection rate. The multiple set collection rate (95\% CI) during the study period was $76.7 \%$ (71.1-82.3\%, $\mathrm{P}<0.05$ ) (Table II). The hospital sizes with the lowest and highest rates were 200-399 beds and $\geq 600$ beds, respectively (Fig. 3D). The multiple set collection rate, excluding pediatric settings $(95 \% \mathrm{CI})$ was $86.2 \%(80.6-91.7 \%, \mathrm{P}<0.05)$ (Table II). In terms of hospital size, the multiple set collection rate increased proportionally with the number of beds (Fig. 3E).
Contamination rate. The contamination rate $(95 \% \mathrm{CI})$ during the study period was $3.1 \%(2.4-3.8 \%, \mathrm{P}<0.05)$ (Table II). In terms of the hospital size, the mean contamination rate decreased as the number of beds increased (Fig. 3F). With the use of antiseptics prior to blood collection, the mean contamination rate was high with PVP-I and low with CHG-AL (Fig. 3G).

\section{Discussion}

In 63 Japanese hospitals, it was found that the positive blood culture rate, number of sets per 1,000 patient days, multiple set collection rate and contamination rate $(95 \% \mathrm{CI})$ were $15.4 \%$ (13.7-17.1\%), 21.5 (18.2-24.9), 76.7\% (71.1-82.3\%) and $3.1 \%$ (2.4-3.8\%), respectively.

Although a positive blood culture rate of $5-15 \%$ is considered appropriate (3), the rate in the present study exceeded $15 \%$. In 2019, the J-SIPHE found a positive blood culture rate $(n=276)$ of $13.3 \%$, which was lower than that in the present study (10), suggesting that the positive rate exceeded $15 \%$ in the present study for specific reasons. This was affected by the fact that there were two facilities which were unique compared 
Table II. Basic data of participating hospitals.

\begin{tabular}{|c|c|c|c|c|c|c|c|c|c|}
\hline \multirow[b]{2}{*}{ Characteristic } & \multicolumn{3}{|c|}{2017} & \multicolumn{3}{|c|}{2018} & \multicolumn{3}{|c|}{ Total } \\
\hline & Value & $95 \% \mathrm{CI}$ & P-value & Value & $95 \% \mathrm{CI}$ & P-value & Value & $95 \% \mathrm{CI}$ & P-value \\
\hline Blood culture positive rate $\%$ & 15.2 & $13.5-16.9$ & $<0.01$ & 15.6 & $13.8-17.5$ & $<0.01$ & 15.4 & $13.7-17.1$ & $<0.01$ \\
\hline \multicolumn{10}{|l|}{ No. of beds } \\
\hline 200-399 & 17.1 & $13.5-20.6$ & $<0.01$ & 17.9 & $14.0-21.7$ & $<0.01$ & 17.3 & $13.8-20.9$ & $<0.01$ \\
\hline $400-499$ & 13.7 & $10.9-16.5$ & $<0.01$ & 14.0 & $11.1-17.0$ & $<0.01$ & 13.9 & $11.1-16.6$ & $<0.01$ \\
\hline $500-599$ & 13.8 & $11.3-16.4$ & $<0.01$ & 13.7 & $10.7-16.7$ & $<0.01$ & 13.7 & $11.0-16.5$ & $<0.01$ \\
\hline$>600$ & 13.9 & $11.8-15.9$ & $<0.01$ & 14.1 & $12.0-16.2$ & $<0.01$ & 14.0 & $12.0-16.0$ & $<0.01$ \\
\hline \multicolumn{10}{|l|}{$\begin{array}{l}\text { Blood culture system } \\
\text { manufacturer }\end{array}$} \\
\hline BD Biosciences & 15.9 & 13.4-18.4 & $<0.01$ & 16.7 & $14.1-19.3$ & $<0.01$ & 16.2 & $13.7-18.7$ & $<0.01$ \\
\hline bioMérieux & 13.3 & $11.1-15.5$ & $<0.01$ & 13.3 & $10.9-15.6$ & $<0.01$ & 13.3 & $11.0-15.5$ & $<0.01$ \\
\hline Beckman Coulter, Inc. & 13.6 & 4.6-22.6 & 0.02 & 13.1 & 7.9-18.4 & $<0.01$ & 13.0 & 7.1-18.9 & $<0.01$ \\
\hline Others & 19.1 & $13.0-25.1$ & $<0.01$ & 17.1 & $-30.0-64.1$ & 0.14 & 19.6 & $14.5-24.7$ & $<0.01$ \\
\hline $\begin{array}{l}\text { Blood culture sampling number } \\
\text { of sets per } 1,000 \text { patient days }\end{array}$ & 20.8 & $17.4-24.1$ & $<0.01$ & 22.3 & $18.9-25.8$ & $<0.01$ & 21.5 & $18.2-24.9$ & $<0.01$ \\
\hline \multicolumn{10}{|l|}{$\begin{array}{l}\text { Total number of hospitalized } \\
\text { patients }\end{array}$} \\
\hline$<100,000$ & 10.6 & $6.7-14.4$ & $<0.01$ & 11.5 & $7.7-15.3$ & $<0.01$ & 11.3 & 7.3-15.2 & $<0.01$ \\
\hline $100,000-149,999$ & 19.7 & $14.0-25.4$ & $<0.01$ & 23.5 & $17.7-29.2$ & $<0.01$ & 20.3 & $14.6-25.9$ & $<0.01$ \\
\hline $150,000-199,999$ & 28.4 & $23.1-33.7$ & 0.02 & 29.6 & $23.9-35.3$ & 0.09 & 29.5 & $24.2-34.8$ & $<0.01$ \\
\hline$\geq 200,000$ & 34.1 & $23.0-45.2$ & 0.15 & 36.2 & $24.8-47.7$ & 0.29 & 35.2 & $24.0-46.4$ & $<0.01$ \\
\hline $\begin{array}{l}\text { Multiple sets rate } \% \\
\text { No. of beds }\end{array}$ & 75.6 & $69.8-81.4$ & $<0.01$ & 77.5 & $71.9-83.2$ & $<0.01$ & 76.7 & $71.1-82.3$ & $<0.01$ \\
\hline $200-399$ & 70.5 & $59.1-82.0$ & $<0.01$ & 72.4 & $61.0-83.8$ & $<0.01$ & 71.7 & $60.5-83.0$ & $<0.01$ \\
\hline $400-499$ & 77.4 & $63.3-91.5$ & $<0.01$ & 80.6 & $67.8-93.4$ & $<0.01$ & 79.1 & $65.8-92.4$ & $<0.01$ \\
\hline $500-599$ & 75.7 & $65.8-85.5$ & $<0.01$ & 76.5 & $66.9-86.1$ & $<0.01$ & 76.1 & $66.5-85.7$ & $<0.01$ \\
\hline$>600$ & 85.1 & 78.9-91.3 & $<0.01$ & 87.0 & $81.1-92.9$ & $<0.01$ & 86.1 & $80.1-92.1$ & $<0.01$ \\
\hline $\begin{array}{l}\text { Multiple sets rate of excluding } \\
\text { pediatrics } \%\end{array}$ & 85.3 & $79.8-90.9$ & $<0.01$ & 86.8 & $81.2-92.3$ & $<0.01$ & 86.2 & $80.6-91.7$ & $<0.01$ \\
\hline \multicolumn{10}{|l|}{ Number of beds } \\
\hline $200-399$ & 78.5 & $67.3-89.7$ & $<0.01$ & 79.5 & $68.0-91.1$ & $<0.01$ & 79.2 & $67.9-90.5$ & $<0.01$ \\
\hline $400-499$ & 85.1 & $70.3-99.8$ & $<0.01$ & 87.8 & $75.0-100.6$ & $<0.01$ & 86.5 & 72.9-100.1 & $<0.01$ \\
\hline $500-599$ & 92.1 & $87.0-97.2$ & $<0.01$ & 93.1 & $88.4-97.7$ & $<0.01$ & 92.6 & $87.7-97.5$ & $<0.01$ \\
\hline$>600$ & 94.3 & $91.4-97.2$ & $<0.01$ & 95.7 & $93.7-97.8$ & $<0.01$ & 95.0 & $92.6-97.5$ & $<0.01$ \\
\hline Contamination rate $\%$ & 3.2 & $2.6-3.8$ & $<0.01$ & 2.9 & $2.2-3.6$ & $<0.01$ & 3.1 & $2.4-3.8$ & $<0.01$ \\
\hline $200-399$ & 3.9 & $2.7-5.1$ & $<0.01$ & 3.3 & $1.9-4.6$ & $<0.01$ & 3.7 & $2.4-5.0$ & $<0.01$ \\
\hline $400-499$ & 2.9 & $1.3-4.6$ & $<0.01$ & 3.1 & $0.8-5.4$ & $<0.01$ & 3.0 & $1.0-5.0$ & $<0.01$ \\
\hline $500-599$ & 2.7 & $1.5-3.8$ & $<0.01$ & 2.3 & $1.3-3.4$ & $<0.01$ & 2.5 & $1.4-3.6$ & $<0.01$ \\
\hline$>600$ & 2.5 & $1.6-3.4$ & $<0.01$ & 2.5 & $1.5-3.6$ & $<0.01$ & 2.6 & $1.6-3.5$ & $<0.01$ \\
\hline \multicolumn{10}{|l|}{ Skin antiseptics used } \\
\hline $0.5 \%$ CHG-AL & 2.1 & $-3.0-7.2$ & 0.12 & 2.3 & $-3.5-8.0$ & 0.01 & 2.2 & $-3.6-7.9$ & 0.13 \\
\hline $1 \%$ CHG-AL & 2.2 & $0.9-3.4$ & $<0.01$ & 2.0 & $1.0-3.0$ & $<0.01$ & 2.2 & $1.0-3.4$ & $<0.01$ \\
\hline 10\% PVP-I & 3.8 & $2.8-4.7$ & $<0.01$ & 3.4 & $2.2-4.5$ & $<0.01$ & 3.8 & $2.7-4.8$ & $<0.01$ \\
\hline $\mathrm{AL}+10 \%$ PVP-I & 4.3 & $-2.3-10.9$ & 0.13 & 5.4 & $-4.1-15.0$ & 0.17 & 4.9 & $-3.2-12.9$ & 0.15 \\
\hline $\mathrm{AL}$ & 2.8 & $0.7-4.9$ & 0.02 & 2.2 & $0.1-4.4$ & 0.04 & 2.5 & $0.4-4.5$ & 0.03 \\
\hline Others & 2.9 & $0.7-5.0$ & 0.03 & 2.9 & $-0.7-6.5$ & 0.08 & 2.9 & $0.0-5.8$ & 0.05 \\
\hline
\end{tabular}

CHG-AL, chlorhexidine gluconate alcohol; PVP-I, povidone iodine; AL + 10\% PVP-I, 70\% alcohol and 10\% povidone iodine; AL, alcohol; CI, confidence interval. 
with the others (top 2 grey dots indicated in the blood culture positive rate illustrated in Fig. 2), with positive blood culture rates of 52.0 and $31.9 \%$ (with the total number of collected sets of 148 and 1,129, respectively). This was likely due to the fact that the total number of collected sets of blood cultures in these facilities was small. Additionally, of the eight facilities with a positive blood culture rate of $>20 \%$, three also had a contamination rate of $>10 \%$, suggesting that frequent contamination may have increased positive rates. Furthermore, 29 of the 63 facilities had a positive blood culture rate of over $15 \%$, indicating that many facilities excessively limited the number of patients for whom blood cultures were performed.

As for positive blood culture rates by blood culture test system manufacturer (a secondary endpoint), only BD Biosciences (16.2\%) was associated with a rate $>15 \%$. This high positive rate occurred as the two facilities with a blood culture positive rate $>30 \%$ (top 2 grey dots indicated in the blood culture positive rate illustrated in Fig. 2.) used BD Biosciences blood culture test systems (when these two facilities were excluded, the positive rate with BD Biosciences decreased to $14.8 \%$ ). However, even when these two facilities were excluded, the positive blood culture rate with BD Biosciences test systems remained higher than the rates for test systems of other manufacturers. In a Chinese study that compared positive blood culture rates among blood culture systems manufactured by BD Biosciences, bioMérieux and Beckman Coulter, Inc., antibiotic treatment prior to blood collection resulted in a higher positive rate with the BD Biosciences system than with the other two systems (15). The present study may also have included cases in which antibiotic agents were administered prior to blood collection, indicating that the differences in positive rates among systems may have included cases in which antibiotics were administered prior to blood collection.

American guidelines recommend 103-188 sets per 1,000 patient days (3). Here, the mean number (21.5) was considerably lower than the recommended range. However, in Europe, the number of sets per 1,000 patient days varies widely by country, from 5.3 to 206.3 (16). Moreover, the number of sets per 1,000 patient days is affected by the mean length of hospital duration, thereby precluding simple comparisons. In the $2019 \mathrm{~J}$-SIPHE survey of 255 healthcare facilities, the median number of sets per 1,000 patient days was 23.8 (10). However, the number of sets per 1,000 patient days in the present study was lower than the reference value. Infectious endocarditis and other severe infections require blood culture tests to assess the progress of treatment, which necessarily leads to a large number of collections (17). Patients with severe infections are often treated in hospitals with a higher number of beds (total number of hospitalized patients), such as core hospitals and university hospitals. Herein, the number of sets increased proportionally with the total number of hospitalized patients. In fact, a number of the facilities exhibited a low total number of hospitalized patients, which may have reduced the overall number of sets per 1,000 patient days.

In a fiscal 2019 report by the All-Japan Hospital Association, the rate of blood culture implementation upon the administration of broad-spectrum antibiotics in 27 facilities was 26.5\% (8). In the Japan Hospital Association's Quality Indicator Project 2018, the mean blood culture implementation rate across 355 facilities was 34.5\% (9), suggesting a disconcerting situation in which Japanese healthcare facilities do not sufficiently implement blood culture, despite the fundamental need to do so. In Europe, the surveillance of blood culture indicators has been conducted in all countries (16). In Japan, it is hoped that J-SIPHE will construct a nationwide surveillance system, conduct analyses by hospital size and enact other improvements to assess the quality of blood culture.

In general, when blood is collected, multiple sets should be collected (18). The multiple set collection rate indicates the suitability of blood collection and should therefore be as close to $100 \%$ as possible. However, in the diagnosis of infective endocarditis and the assessment of treatment, solitary sets are sometimes collected for several consecutive days; thus, cases of $100 \%$ multiple set collection are rare in clinical practice. In the Japan Hospital Association's Quality Indicator Project 2018, the multiple set collection rate across 355 healthcare facilities was $60.4 \%$ (9); the present study had a higher rate $(76.7 \%$ ). Although the reason for the high multiple set collection rate cannot be clearly identified, the number of participating facilities and differences in the methods of calculating multiple set collection rates (the numerator in the formula) may have played a role.

A multiple set collection rate of $10-19 \%$ has been reported in a Japanese pediatric clinical setting (19). Ensuring that a sufficient volume of blood is collected from children poses an issue, and multiple sets of collections have not progressed in pediatric settings. In 2019, J-SIPHE reported a rather low multiple set collection rate of $4.9 \%$ in children aged $<15$ years at 178 Japanese healthcare facilities (10). In the present study, the multiple set collection rate increased by $\sim 10 \%$ when children were excluded. Therefore, the assessments of multiple set collection rates should consider special circumstances in pediatric settings.

Blood culture contamination, a phenomenon in which microorganisms normally not present in blood are detected in blood culture (20), is highly likely to lead to inappropriate antibiotic treatments and needs to be prevented as much as possible. In a 1998 American study, a 2009 study including six Japanese healthcare facilities and the 2019 J-SIPHE report $(n=276)$, the contamination rates were $2.5 \%(21), 1.8 \%(6)$ and $1.4 \%$ (10), respectively. The contamination rate in the present study was $3.1 \%$, which was higher than that reported in previous studies. The higher contamination rate in the present study was likely due to differences in medical backgrounds. In the USA, blood collection specialist teams are often used; the two Japanese studies involved only medium- and large-sized hospital, and the contamination rate in the present study decreased to $2.5 \%$ when limited to hospitals with $\geq 500$ beds. The high contamination rate in small hospitals obtained in the present study may be due to the fact that small hospitals often do not have clinical laboratory technicians or other staff familiar with blood culture, and therefore lack education in appropriate blood collection.

As regards the association between antiseptics used prior to blood collection (a secondary endpoint) and the contamination rate, the contamination rates were lower with $\mathrm{CHG}-\mathrm{AL}$ and $\mathrm{AL}$ than with PVP-I. A 2011 meta-analysis reported that the blood culture contamination rate was $67 \%$ lower with $\mathrm{CHG}-\mathrm{AL}$ than with PVP-I (22). Although PVP-I was associated with a high 
contamination rate, it was used by $>40 \%$ of facilities examined in the present study. This concerning frequent use of PVP-I could lead to incorrect antibiotic treatment.

A limitation of the present study is that it is unclear whether the results reflect the overall population due to the small number of samples, with only 63 participating facilities. Furthermore, the distribution of participating hospitals suggests a high participation rate in densely populated areas, and the accumulation of additional data is warranted for the generalization of the results. In addition, as facilities with $<200$ beds were not eligible for participation in the study, healthcare facilities could not be sampled from a population throughout Japan. Furthermore, as the characteristics of medical care in each healthcare facility (such as stratified evaluation of clinical departments) were not analyzed, a bias is expected in the positive blood culture rate specific to clinical departments.

Despite such a bias in the present study, the results of the four indicators $(95 \% \mathrm{CI})$ may be used as reference values for blood cultures in healthcare facilities with $\geq 200$ beds in Japan, which may guide the assessment of the quality of blood culture in each facility. The authors also aim to approach these values in a hospital and hope that on-site interventions and educational activities will be used to improve the state of blood culture at apiece hospital as much as possible.

\section{Acknowledgements}

The authors would like to express their deep gratitude to the following individuals for the provision of data for the study: Ms. Sakiko Igawa of JCHO Gunma Chuo Hospital, Mr. Yudai Watanabe of Asahi Chuo Hospital, Ms. Kumiko Okuda of Ichinomiyanishi Hospital, Ms. Chisumi Mukai of Iwate Prefectural Central Hospital, Dr Kenji Yamamoto of Utano National Hospital, Mr. Tadashi Ohno of Omuta Tenryo Hospital, Mr. Masaaki Sasano of Okazaki City Hospital, Ms. Miwaka Tomo of Kaisei Hospital, Mr. Kazuki Niwa of Kanto Rosai Hospital, Mr. Atsushi Yasuda of Kitami Red Cross Hospital, Ms. Akiko Iwama of Kimitsu Chuo Hospital, Mr. Tomohiro Hamashima of Kumamoto Rosai Hospital, Ms. Masami Fujita of Kurashiki Heisei Hospital, Ms. Yoriko Ueno of Kurihara Central Hospital, Ms. Saki Matsumoto of Konan Medical Center; Mr. Tatsuya Uchida of Obama Municipal Hospital, Ms. Momoyo Miyata of International University of Health and Welfare Hospital, Mr. Takeshi Ogino of Saiseikai Kawaguchi General Hospital, Ms. Chieko Tsuji of Saiseikai Kurihashi Hospital, Mr. Kosuke Yasui of Saiseikai Chuwa Hospital, Ms. Yuri Shoji of Saiseikai Yamagata Saisei Hospital, Ms. Mayumi Takase of Saiseikai Maebashi Hospital, Ms. Kiyoko Yamamoto of Saiseikai Yamaguchi General Hospital, Ms. Akane Kamiya of Saiseikai Yokohamashi Nanbu Hospital, Mr. Shinzo Kawasaki of Saiseikai Wakayama Hospital, Ms. Tatsuko Okuda of Saiseikai Hiroshima Hospital, Ms. Yukari Sano of Saga-ken Medical Centre Koseikan, Ms. Kana Fujita of Asahikawa City Hospital, Mr. Kazumasa Koga of Tamagawa Hospital,Ms. Tomoko Fudeyasu of Tsuyama Chuo Hospital, Ms. Hisae Fukuyama of The Fraternity Memorial Hospital, Mr. Tetsuyoshi Taneichi and Ms. Yuki Matsubara of Tokatsu Hospital, Mr. Masami Kurokawa of JCHO Tokyo Shinjuku Medical Center, Ms. Fumi Togashi of Tohoku Kosai Hospital, Ms. Mina Takano of Nagaoka Red
Cross Hospital, Ms. Ayako Yamamoto of Niigata Prefectural Central Hospital, Mr. Yuudai Kanazawa of Hachinohe City Hospital, Mr. Takahiro Suzuki of Hitachi General Hospital, Ms. Kumiko Mandokoro of Fukuoka Mirai Hospital, Dr Kiyohito Ishikawa of Fujita Health University Hospital, Mr. Masakazu Yoshida of Maebashi Red Cross Hospital, Ms. Yurika Notsu of Mizushima Kyodo Hospital, Dr Masaru Amishima MD of NHO Hokkaido Medical Center, Ms. Risa Kato of NHO Niigata National Hospital, Mr. Satoshi Shinomiya of Minoh City Hospital, Ms. Toko Kimura of Yamagata City Hospital Saiseikan, Ms. Tomoko Fujiwara of Yamaguchi Prefectural Medical Center, Ms. Yuka Shimokawa of Yokosuka Kyosai Hospital, and Ms. Mayumi Sato of Yokohama Minami Kyousai Hospital. All affiliations and names were current at the time of participation in the study.

\section{Funding}

The present study was supported by the Social Welfare Organization Saiseikai Imperial Gift Foundation, Inc. Medical/Welfare Joint Subsidy Financing Systems.

\section{Availability of data and materials}

All data generated or analyzed during this study are included in this published article.

\section{Authors' contributions}

TO was the chief investigator and responsible for the data analysis and organization. MO, EK and KI collected the data and confirm the authenticity of all the raw data. All authors contributed to the writing of the final manuscript. All authors have read and approved the final manuscript.

\section{Ethics approval and consent to participate}

The study protocol was approved by the Institutional Review Board of Saiseikai Yokohamashi Tobu Hospital (2018107). Informed consent was not required due to the retrospective nature of the study.

\section{Patient consent for publication}

Not applicable.

\section{Competing interests}

The authors declare that they have no competing interests.

\section{References}

1. Stefani S: Diagnostic techniques in bloodstream infections: Where are we going? Int J Antimicrob Agents 34 (Suppl 4): S9-S12, 2009.

2. Opota O, Croxatto A, Prod'hom G and Greub G: Blood culture-based diagnosis of bacteraemia: State of the art. Clin Microbiol Infect 21: 313-322, 2015.

3. Baron EJ, Weinstein MP, Dunne WM, Yagupsky P, Welch DF and Wilson DM: Cumitech 1C, Blood cultures IV. In: American Society for Microbiology. Baron EJ (ed). ASM Press, Washington, DC, 2005. 
4. Sánchez-Sánchez MM, Arias-Rivera S, Fraile-Gamo P, Jareño-Collado R, López-Román S, Vadillo-Obesso P, García-González S, Pulido-Martos MT, Sánchez-Muñoz EI, Cacho-Calvo J, et al: Effect of a training programme on blood culture contamination rate in critical care. Enferm Intensiva 29 121-127, 2018.

5. Novis DA, Dale JC, Schifman RB, Ruby SG and Walsh MK: Solitary blood cultures: A College of American Pathologists Q-probes study of 132,778 blood culture sets in 333 small hospitals. Arch Pathol Lab Med 125: 1290-1294, 2001.

6. Ohmagari N, Takakura S, Matsumura Y, Sugiyama T, Takeshita N, Takahashi M, Ainoda Y, Goto A, Chibana N, Ohshiro T, et al: A questionnaire survey of blood culture among Japanese hospitals: A pilot study. J Jpn Soc Clin Microbiol 22: 13-19, 2012 (In Japanese)

7. Akemi K, Kazuhiko T, Hidekazu N, Hitomi M, Manami I, Yoshibumi A and Yoshihito O: Difference of the bacteria isolated using BACTEC and BacT/ALERT for automatic blood culture analysis. Jpn J Med Technol 63: 24-28, 2014.

8. All-Japan Hospital Association: Fiscal 2018 blood culture implementation (blood culture implementation rate). https://www.ajha or.jp/hms/qualityhealthcare/pdf/2019/all/2019all_outcome_55. pdf. Accessed June 23, 2020

9. Japan Hospital Association: Quality Indicator Project 2018. https://www.hospital.or.jp/pdf/06_20191120_01.pdf.

10. National Center for Global Health and Medicine, AMR Clinical Reference Cen ter: Japan Surveillance for Infection Prevention and Healthcare Epidemiology (J-SIPHE) Annual Report 2019. J-SIPHE, Tokyo, 2019. https://j-siphe.ncgm.go.jp/files/JSIPHE_ AnnualReport_2019en.pdf. Accessed June 23,2020.

11. Zadroga R, Williams DN, Gottschall R, Hanson K, Nordberg V, Deike M, Kuskowski M, Carlson L, Nicolau DP, Sutherland C, et al: Comparison of 2 blood culture media shows significant differences in bacterial recovery for patients on antimicrobial therapy. Clin Infect Dis 56: 790-797, 2013.

12. Suwanpimolkul G, Pongkumpai M and Suankratay C: A randomized trial of $2 \%$ chlorhexidine tincture compared with $10 \%$ aqueous povidone-iodine for venipuncture site disinfection: Effects on blood culture contamination rates. J Infect 56: 354-359, 2008

13. Schifman RB, Bachner P and Howanitz PJ: Blood culture quality improvement: A College of American Pathologists Q-Probes study involving 909 institutions and 289,572 blood culture sets. Arch Pathol Lab Med 120: 999-1002, 1996.
14. Kanda Y: Investigation of the freely available easy-to-use software 'EZR' for medical statistics. Bone Marrow Transplant 48: 452-458, 2013

15. Li G, Sun J, Pan S, Li W, Zhang S, Wang Y, Sun X, Xu H and Ming L: Comparison of the performance of three blood culture systems in a Chinese tertiary-care hospital. Front Cell Infect Microbiol 9: 285, 2019.

16. European Centre for Disease Prevention and Control (ECDC): Surveillance of antimicrobial resistance in Europe 2018. ECDC, Stockholm, 2018. https://www.ecdc.europa. $\mathrm{eu} / \mathrm{sites} / \mathrm{default} /$ files/documents/surveillance-antimicrobialresistance-Europe-2018.pdf. Accessed June 23, 2020.

17. Nakatani S, Ohara T, Ashihara K, Izumi C, Iwanaga S, Eishi K, Okita Y, Daimon M, Kimura T, Toyoda K, et al; Japanese Circulation Society Joint Working Group: JCS 2017 Guideline on Prevention and Treatment of Infective Endocarditis. Circ J 83: 1767-1809, 2019.

18. Lee A, Mirrett S, Reller LB and Weinstein MP: Detection of bloodstream infections in adults: How many blood cultures are needed? J Clin Microbiol 45: 3546-3548, 2007.

19. Kasai M, Shime N, Saitou A, Funaki T, Shoji K and Miyairi I: Investigating blood culture collection in a Japanese pediatric clinical setting. Kansenshogaku Zasshi 87: 620-623, 2013 (In Japanese).

20. Hall KK and Lyman JA: Updated review of blood culture contamination. Clin Microbiol Rev 19: 788-802, 2006.

21. Schifman RB, Strand CL, Meier FA and Howanitz PJ: Blood culture contamination: A College of American Pathologists Q-Probes study involving 640 institutions and 497,134 specimens from adult patients. Arch Pathol Lab Med 122: 216-221, 1998.

22. Caldeira D, David C and Sampaio C: Skin antiseptics in venous puncture-site disinfection for prevention of blood culture contamination: Systematic review with meta-analysis. J Hosp Infect 77: 223-232, 2011.

This work is licensed under a Creative Commons Attribution-NonCommercial-NoDerivatives 4.0 International (CC BY-NC-ND 4.0) License. 